\title{
The downregulation of Rap1 GTPase-activating protein is associated with a poor prognosis in colorectal cancer and may impact on tumor progression
}

\author{
WEI-LI GAO, GUO-CHAO YE, LI-WEI LIU and LU WEI \\ Department of General Surgery, Huzhou Central Hospital, Zhejiang University, Huzhou, Zhejiang 313000, P.R. China
}

Received May 23, 2017; Accepted February 15, 2018

DOI: $10.3892 / 01.2018 .8305$

\begin{abstract}
Rap1 GTPase-activating protein(Rap1GAP) has been reported to serve an important role in various types of cancer by specific stimulation as a negative regulator of Rap1 activity. However, the role of Rap1GAP in colorectal cancer (CRC) has yet to be fully elucidated. The aim of the present study was to investigate the expression of Rap1GAP in CRC tissues and to elucidate its clinical significance. The expression of Rap1GAP, matrix metallopeptidase 9 (MMP-9) and E-cadherin in 227 $\mathrm{CRC}$ tissues and paired para-carcinoma tissues was detected by immunohistochemistry. Associations between Rap1GAP expression and clinicopathological characteristics, and between Rap1GAP expression and prognostic value (OS + DFS) in CRC were investigated. Furthermore, associations between Rap1GAP expression and MMP-9 expression, and between Rap1GAP expression and E-cadherin expression were also investigated. Rap1GAP expression was markedly downregulated in CRC tissues compared with para-carcinoma tissues. Decreased expression of Rap1GAP was significantly associated with depth of invasion, lymph node metastasis, advanced Tumor-Node-Metastasis stage and a poor prognosis in patients with CRC following surgery. Furthermore, univariate and multivariate analyses revealed that Rap1GAP was an independent poor prognostic factor for disease-free survival and overall survival. In addition, Rap1GAP expression was negatively associated with MMP-9 and positively associated with E-cadherin in $227 \mathrm{CRC}$ samples. In brief, the results of the present study suggested that Rap1GAP may be involved in tumor progression in CRC and may serve as a potential target for prognostic prediction of patients with CRC.
\end{abstract}

Correspondence to: Dr Wei-Li Gao, Department of General Surgery, Huzhou Central Hospital, Zhejiang University, 198 Hongqi Road, Huzhou, Zhejiang 313000, P.R. China

E-mail: gw10974@163.com

Key words: Rap1 GTPase-activating protein, matrix metallopeptidase 9, E-cadherin, colorectal cancer, prognosis

\section{Introduction}

Colorectal cancer (CRC) is the third most commonly diagnosed malignancy in males and the second in females worldwide (1). Despite improvements in surgical techniques and adjuvant chemotherapy in recent years, CRC remains the fourth leading cause of cancer-associated mortalities throughout the world $(1,2)$. At present, the Tumor-Node-Metastasis (TNM) staging classification is the main tool in the prediction of CRC prognosis; however, clinical outcome differs greatly even among patients with the same TNM stage of disease (3). Therefore, it is necessary to identify novel therapeutic targets and biomarkers for the prognosis of CRC.

Rap1 GTPase-activating protein (Rap1GAP) is a member of a family of GTPase activation proteins, which may work with Rapl-GTP, to make the latter become inactive Rapl-GDP, thereby making Rapl inactivation as a negative regulator (4-7). Previous reports have demonstrated that expression of Rap1GAP was downregulated in various tumor types, including pancreatic cancer (8), thyroid cancer (9-11), head and neck squamous cell carcinoma (12), melanoma (13), acute myeloid leukemia (14), and prostate cancer (15). Furthermore, various in vivo and in vitro studies have suggested that Rap1GAP, as a type of tumor suppressor gene, serves an important role in tumor cell proliferation, invasion, migration and metastasis (10,13,16-18). In addition, decreased expression of Rap1GAP was associated with a poor prognosis in endometrial cancer and gastric cancer $(19,20)$.

The downregulation of Rap1GAP in CRC tumor cells where alterations in cell-cell and cell-matrix adhesion are early steps in tumor dissemination supports a role for Rap1GAP depletion in tumor progression $(18,21)$. However, the role of Rap1GAP in $\mathrm{CRC}$ has not been fully elucidated. Therefore, the aim of the present study was to investigate the expression of Rap1GAP in CRC tissues and to evaluate whether Rap1GAP expression is associated with clinicopathological characteristics and the prognosis of CRC.

\section{Materials and methods}

Patients and specimens. CRC tissue specimens and paired adjacent para-carcinoma mucosa tissues were obtained from 227 patients by surgical resection in the Department of General Surgery, Huzhou Central Hospital (Zhejiang, China) between February 2008 and March 2012. Tissue specimens were 
frozen immediately in liquid nitrogen after surgery and stored at $-80^{\circ} \mathrm{C}$ until use. Then tissues were fixed in $10 \%$ formalin and paraffin-embedded. The mean age of the patients was $56.48 \pm 10.54$ years (range, 21-79 years). There were 131 male and 96 female patients. The criteria for enrollment included the following: i) newly histopathologically diagnosed CRC by; ii) no history of other tumors; iii) no neoadjuvant or adjuvant chemotherapy prior to surgery; iv) no radiotherapy prior to surgery; and v) complete clinicopathological data and available tissue specimens. The exclusion criteria for enrollment were as follows: i) patients who succumbed to mortality within 3 months after surgery; ii) patients with severe heart, lung, liver or kidney disease; and iii) patients who failed to undergo radical resection.

Clinicopathological data are presented in Table I. Cancer staging relies on the TNM system designed by the American Joint Committee on Cancer. The study protocols were approved by the Ethics Committee and the Human Research Review Committee of Huzhou Central Hospital and met the guidelines of the responsible governmental agency. Written informed consent for the use of these samples for research were obtained from the patients or their relatives.

Immunohistochemistry. Immunohistochemical analysis was used to evaluate expression levels in all samples. Serial sections $(4 \mu \mathrm{m})$ from the paraffin-embedded blocks were deparaffinized in xylene and rehydrated in a descending ethanol series. Next, the sections were placed in $0.01 \mathrm{M}$ sodium citrate buffer ( $\mathrm{pH}$ 6.0) and autoclaved at $121^{\circ} \mathrm{C}$ for $10 \mathrm{~min}$ for antigen retrieval. After $30 \mathrm{~min}$ of pre-incubation in 3\% normal goat serum to prevent non-specific staining at room temperature, the sections were incubated with a primary antibody against Rap1GAP (dilution, 1:200; cat. no. sc-166586; Santa Cruz Biotechnology, Inc., Dallas, TX, USA) at $4^{\circ} \mathrm{C}$ overnight. The sections were subsequently treated using the streptavidin-peroxidase technique (OriGene Technologies, Inc., Beijing, China), according to the manufacturer's protocols. Negative controls were made by the omission of the primary antibody during the procedure of immunohistochemistry. Primary antibodies against MMP-9 (dilution, 1:200; cat. no. ab119906; Abcam, Cambridge, UK) and E-cadherin (dilution, 1:200; cat. no. ab76055; Abcam) were also used for immunohistochemical staining at $4^{\circ} \mathrm{C}$ overnight. A 30-min incubation at room temperature with a peroxidase-conjugated affinity purified goat anti-mouse IgG secondary antibody (dilution, 1:200; cat. no. TA130003 OriGene Technologies, Inc.) followed.

Evaluation of immunohistochemical analysis. Two pathologists blinded to the clinical features of the patients with CRC independently examined the sections and were in agreement with the final effective immunostaining under a light microscope at $\mathrm{x} 100, \mathrm{x} 200$ or $\mathrm{x} 400$ magnification. The intensity of immunostaining and the percentage of positive tumor cells were taken into consideration when analyzing the final quantitation of each staining. The staining was scored by multiplying the following two scores: i) the staining intensity $(0$, negative; 1 , weak; 2 , moderate; and 3 , high) and ii) the percentage of positive cells $(0, \leq 5 \%$ tumor cells with staining; $1,6-20 \%$ tumor cells with staining; $2,21-40 \%$ tumor cells with staining; 3,41-60\% tumor cells with staining; $4,61-80 \%$ tumor cells with staining; and 5, >81\% tumor cells with staining). Rap1GAP and E-cadherin expression was defined as decreased when the score was $\leq 6$, and expression was defined as increased when the score was $>6$. The tumors were regarded as having a high expression of MMP-9 when the tumor sample reached a score of $\geq 8$, and tumor samples with a score of $<8$ were considered as having a low expression of MMP-9.

Follow-up. The follow-up deadline was December 2016. All patients were followed up regularly every 3 months for the first 2 years after surgery, every 6 months until the fifth year, and then annually thereafter. Overall survival (OS) was defined as the interval between the date of surgery and the date that of patient mortality from CRC or the follow-up deadline. Recurrence were diagnosed by tumor biomarkers levels, including carcinoembryonic antigen (CEA), ultrasonic examination and computed tomography (CT) or magnetic resonance imaging (MRI) during the follow-up period. Disease-free survival (DFS) was defined as the interval between the day of surgery and the date of CRC recurrence. If recurrence was not confirmed, the date of mortality due to CRC or the follow-up deadline was used.

Statistical analysis. All data were processed using SPSS version 17.0 statistical software (SPSS, Inc., Chicago, IL, USA). Differences between two groups were compared using Student's t-tests. The association between Rap1GAP expression and the patient clinicopathological characteristics were analyzed using a $\chi^{2}$ test. DFS and OS curves were calculated using the Kaplan-Meier method and intergroup differences were examined using the log-rank test. The prognostic factors were determined by DFS and OS analysis using the univariate and multivariate Cox proportional hazards model. The association between the Rap1GAP expression and expression of MMP-9 and E-cadherin was analyzed by the Spearman's rank correlation coefficient. $\mathrm{P}<0.05$ was considered to indicate a statistically significant difference.

\section{Results}

Decreased expression of RaplGAP and E-cadherin, and increased expression of MMP-9 in CRC. The expression of RaplGAP was detected by immunohistochemistry in 227 colorectal adenocarcinoma and paired adjacent para-carcinoma tissues. As demonstrated in Fig. 1A, RaplGAP was mainly located in the cytoplasm of the normal follicular epithelial cells. Additionally, RaplGAP expression levels were markedly lower in CRC than in adjacent para-carcinoma tissues $(\mathrm{P}<0.001$; Fig. 1B). Furthermore, a significantly decreased expression of RaplGAP was present in $62.1 \%$ (141/227) of CRC tissues compared with $36.1 \%(82 / 227)$ of adjacent para-carcinoma tissues $(\mathrm{P}<0.001$; Fig. 1C).

The expression of MMP-9 and E-cadherin in 227 colorectal adenocarcinoma tissues was determined by immunohistochemistry. As a type of aberrant metastasis protein, MMP-9 was more highly expressed in CRC tissues compared with expression in adjacent para-carcinoma tissues, which was observed mainly in the cytoplasm (Fig. 1). The expression of E-cadherin as the epithelial protein was lower in the CRC tissues than that in adjacent para-carcinoma tissues (Fig. 1). 
Table I. Association between Rap1GAP expression and clinicopathological characteristics of patients with colorectal cancer.

\begin{tabular}{|c|c|c|c|c|}
\hline \multirow[b]{2}{*}{ Characteristic } & \multirow[b]{2}{*}{ No. patients } & \multicolumn{2}{|c|}{ Rap1GAP expression } & \multirow[b]{2}{*}{ P-value } \\
\hline & & Low $(\%)$ & High (\%) & \\
\hline Sex & & & & 0.542 \\
\hline Male & 131 & $83(63.4)$ & 48 (36.6) & \\
\hline Female & 96 & $57(59.4)$ & $39(40.6)$ & \\
\hline Age, years & & & & 0.215 \\
\hline$<60$ & 103 & $59(57.3)$ & $44(42.7)$ & \\
\hline$\geq 60$ & 124 & $81(65.3)$ & $43(34.7)$ & \\
\hline Diameter of tumor, $\mathrm{cm}$ & & & & 0.060 \\
\hline$<5$ & 123 & $69(56.1)$ & $54(43.9)$ & \\
\hline$\geq 5$ & 104 & $71(68.3)$ & $33(31.7)$ & \\
\hline Differentiation & & & & 0.131 \\
\hline Well & 62 & $36(58.1)$ & $26(41.9)$ & \\
\hline Moderate & 76 & $42(55.3)$ & $34(44.7)$ & \\
\hline Poor & 89 & $62(69.7)$ & $27(30.3)$ & \\
\hline Tumor location & & & & 0.832 \\
\hline Colon & 109 & $68(62.4)$ & 41 (37.6) & \\
\hline Rectum & 118 & $72(61.0)$ & $46(39.0)$ & \\
\hline Depth of invasion & & & & 0.015 \\
\hline $\mathrm{T} 1+\mathrm{T} 2$ & 51 & $24(47.1)$ & $27(52.9)$ & \\
\hline $\mathrm{T} 3+\mathrm{T} 4$ & 176 & $116(65.9)$ & $60(34.1)$ & \\
\hline Lymph node metastasis & & & & 0.002 \\
\hline No & 93 & 46 (49.5) & $47(50.5)$ & \\
\hline Yes & 134 & $94(70.1)$ & $40(29.9)$ & \\
\hline TNM stage & & & & 0.004 \\
\hline I & 37 & $16(43.2)$ & $21(56.8)$ & \\
\hline II & 56 & 37 (66.1) & $19(33.9)$ & \\
\hline III & 134 & 97 (72.4) & 37 (27.6) & \\
\hline
\end{tabular}

Rap1GAP, Rap1 GTPase-activating protein; TNM, tumor-node-metastasis.

Decreased expression of RaplGAP is correlated with certain clinicopathological characteristics in CRC. To elucidate the clinical significance of RaplGAP expression in CRC, the associations between RaplGAP expression and clinicopathological characteristics in 227 CRC samples were analyzed. As demonstrated in Table I, there were no correlations between the expression of RaplGAP and sex, age, diameter of tumor, histological differentiation or tumor location (all $\mathrm{P}>0.05$ ). However, the decreased expression of RaplGAP was significantly associated with the depth of invasion $(\mathrm{P}=0.015)$, lymph node metastasis $(\mathrm{P}=0.002)$ and advanced TNM stage $(\mathrm{P}=0.004)$. Notably, the significant negative correlations between RaplGAP expression and serosal invasion or lymph node metastasis implied a potential role of RaplGAP in enhancing the invasion and metastasis of CRC.

Decreased expression of RaplGAP impacts on tumor progression in $C R C$. In order to further elucidate the role of RaplGAP expression on the aggressiveness of CRC, the associations between RaplGAP expression and the depth of invasion or lymph node metastasis were analyzed. The rates of RaplGAP low-expression were 65.9 and $70.1 \%$ in the T3+T4 group and N1-3 group, whereas they were only 47.1 and $49.5 \%$ in $\mathrm{T} 1+\mathrm{T} 2$ and $\mathrm{N} 0$ groups $(\mathrm{P}=0.015$ and $\mathrm{P}=0.002$, respectively; Table I). Furthermore, the expression levels of RaplGAP were lower in the T3+T4 and N1-3 groups than in the $\mathrm{T} 1+\mathrm{T} 2$ and $\mathrm{N} 0$ groups $(\mathrm{P}=0.001$ and $\mathrm{P}=0.003$, respectively; Fig. 2). Additionally, in order to investigate whether decreased expression of RaplGAP accounts for its invasion and metastasis features in CRC, the present study also evaluated the associations between RaplGAP expression and the expression of MMP-9 and E-cadherin in 227 CRC samples. Notably, the decreased expression of RaplGAP was negatively correlated with MMP-9 expression ( $\mathrm{r}=-0.444$, $\mathrm{P}<0.001$; Fig. $3 \mathrm{~A}$ ) and positively associated with E-cadherin expression ( $\mathrm{r}=0.573$, $\mathrm{P}<0.001$; Fig. 3B).

Decreased expression of RaplGAP is associated with poor prognosis in CRC. Kaplan-Meier analysis indicated that the DFS and OS rates of the decreased expression of Rap1GAP 
Table II. Univariate and multivariate analyses of prognostic factors for disease-free survival or overall survival of patients with colorectal cancer.

\begin{tabular}{|c|c|c|c|c|c|c|}
\hline \multirow[b]{2}{*}{ Variable } & \multicolumn{3}{|c|}{ Univariate analysis } & \multicolumn{3}{|c|}{ Multivariate analysis } \\
\hline & HR & $95 \% \mathrm{CI}$ & P-value & HR & $95 \% \mathrm{CI}$ & P-value \\
\hline \multicolumn{7}{|l|}{ Disease-free survival } \\
\hline Rap1GAP expression (low/high) & 1.944 & $1.330-2.842$ & 0.001 & 1.738 & $1.179-2.560$ & 0.005 \\
\hline Sex (female/male) & 1.020 & $0.720-1.446$ & 0.911 & & & \\
\hline Age $(\geq 60 /<60$ years $)$ & 1.298 & $0.914-1.843$ & 0.146 & & & \\
\hline Diameter of tumor, $\mathrm{cm}(\geq 5 /<5)$ & 1.331 & $0.942-1.879$ & 0.105 & & & \\
\hline Differentiation (poor/well+moderate) & 1.366 & $0.965-1.934$ & 0.079 & & & \\
\hline Tumor location (rectum/colon) & 1.223 & 0.864-1.732 & 0.256 & & & \\
\hline Depth of invasion $(\mathrm{T} 3+\mathrm{T} 4 / \mathrm{T} 1+\mathrm{T} 2)$ & 2.039 & $1.277-3.257$ & 0.003 & 1.576 & $0.951-2.613$ & 0.078 \\
\hline Lymph node metastasis (yes/no) & 2.000 & $1.376-2.907$ & $<0.001$ & 1.640 & $1.094-2.458$ & 0.017 \\
\hline TNM stage $(\mathrm{III} / \mathrm{I}+\mathrm{II})$ & 1.686 & $1.173-2.424$ & 0.005 & 1.491 & $1.031-2.157$ & 0.034 \\
\hline \multicolumn{7}{|l|}{ Overall survival } \\
\hline Rap1GAP expression (low/high) & 1.937 & $1.284-2.921$ & 0.002 & 1.672 & $1.099-2.544$ & 0.016 \\
\hline Sex (female/male) & 1.002 & $0.688-1.458$ & 0.993 & & & \\
\hline Age $(\geq 60 /<60$ years $)$ & 1.321 & $0.905-1.927$ & 0.149 & & & \\
\hline Diameter of tumor, $\mathrm{cm}(\geq 5 /<5)$ & 1.328 & $0.954-2.002$ & 0.087 & & & \\
\hline Differentiation (poor/well+moderate) & 1.319 & $0.908-1.916$ & 0.146 & & & \\
\hline Tumor location (rectum/colon) & 1.265 & $0.870-1.840$ & 0.218 & & & \\
\hline Depth of invasion $(\mathrm{T} 3+\mathrm{T} 4 / \mathrm{T} 1+\mathrm{T} 2)$ & 2.139 & $1.275-3.586$ & 0.004 & 1.705 & $0.980-2.966$ & 0.059 \\
\hline Lymph node metastasis (yes/no) & 1.886 & $1.264-2.814$ & 0.002 & 1.484 & $0.965-2.283$ & 0.072 \\
\hline TNM stage (III/I+II) & 1.787 & $1.205-2.651$ & 0.004 & 1.596 & $1.069-2.382$ & 0.022 \\
\hline
\end{tabular}

Variables with $\mathrm{P}<0.05$ in univariate analysis were included in the multivariate analysis. Rap1GAP, Rap1 GTPase-activating protein; HR, hazard ratio; CI, confidence interval; TNM, tumor-node-metastasis.

group were significantly poorer than that of the increased expression of Rap1GAP group $(\mathrm{P}<0.001$ and $\mathrm{P}=0.001$, respectively; Fig. 4A). In addition, the depth of invasion $(\mathrm{P}=0.002$ and $\mathrm{P}=0.003$, respectively; Fig. $4 \mathrm{~B}$ ), lymph node metastasis $(\mathrm{P}<0.001$ and $\mathrm{P}=0.001$, respectively; Fig. $4 \mathrm{C})$ and advanced TNM stage $(\mathrm{P}=0.004$ and $\mathrm{P}=0.003$, respectively; Fig. 4D) were markedly associated with poorer DFS and OS rates, as determined by Kaplan-Meier analysis. Furthermore, multivariate analysis suggested that decreased expression of Rap1GAP was an independent prognostic factor for DFS and OS $(\mathrm{P}=0.005$ and $\mathrm{P}=0.016$, respectively; Table II). Multivariate analysis also revealed that an advanced TNM stage was an independent predictive factor for DFS and $\mathrm{OS}(\mathrm{P}=0.034$ and $\mathrm{P}=0.022$, respectively), while lymph node metastasis only was an independent prognostic factor for DFS $(\mathrm{P}=0.017$; Table II).

\section{Discussion}

In the present study, decreased expression of Rap1GAP was correlated with tumor progression and poor prognosis in CRC. To the best of our knowledge, the present study is the first to report the clinical significance of Rap1GAP expression in CRC.

Rap1GAP is a member of the GTPase-activation protein family, which promotes the hydrolysis of GTP to GDP by specific stimulation as a negative regulator of Rap1 activity (4-7). The present study revealed that Rap1GAP expression was lower in CRC compared with adjacent para-carcinoma tissues by immunohistochemical examination. Furthermore, decreased expression of Rap1GAP was correlated with certain clinicopathological outcomes, including the depth of invasion, lymph node metastasis and advanced TNM stages in CRC. Additionally, the patients with low Rap1GAP expression had significantly poorer prognoses compared with the patients with high Rap1GAP expression, as determined using Kaplan-Meier survival analysis. The multivariate Cox proportional hazards model analysis revealed that decreased expression of Rap1GAP was an independent factor for a poorer prognosis (DFS and OS) in CRC. These observations were consistent with previously reported results. Decreased expression of Rap1GAP has been reported in pancreatic cancer (8), thyroid cancer (9-11), head and neck squamous cell carcinoma (12), melanoma (13), acute myeloid leukemia (14) and gastric cancer (20). Furthermore, the low expression of Rap1GAP in gastric cancer was significantly associated with TNM stage, lymph node metastasis, tumor diameter and poor OS, and therefore may be an independent prognostic factor of gastric cancer (20). These results strongly indicated that Rap1GAP may be used to identify individual patients with $\mathrm{CRC}$ who have a higher risk of recurrence and mortality and are therefore good candidates for receiving more aggressive treatment. 
A

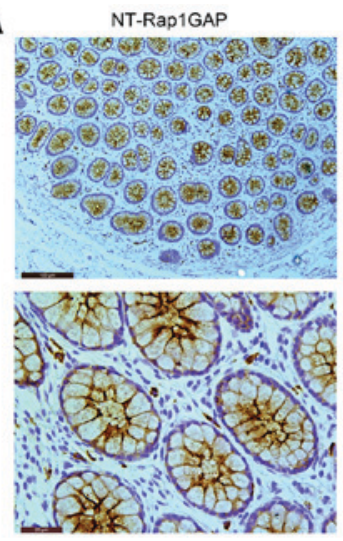

T-Rap1GAP
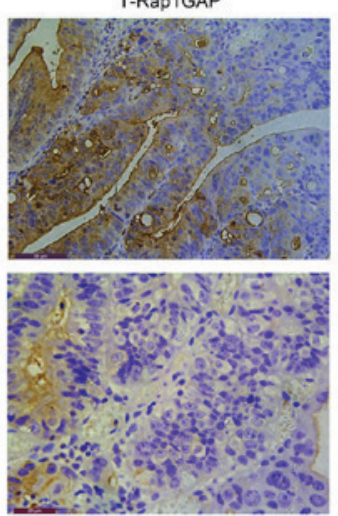

T-MMP-9
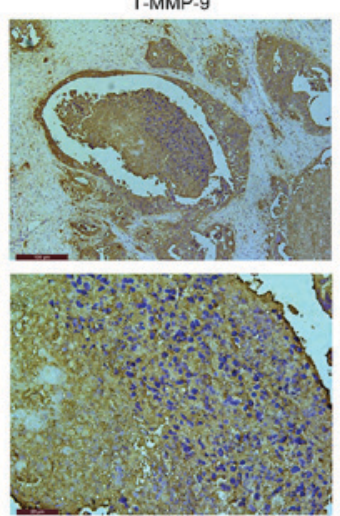

T-E-cadherin
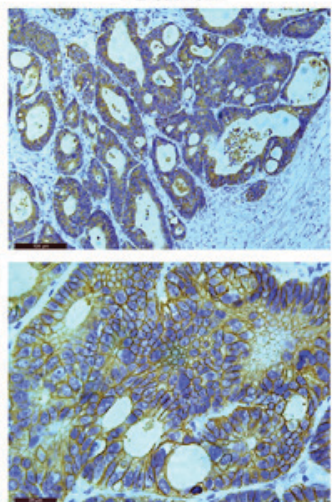

B
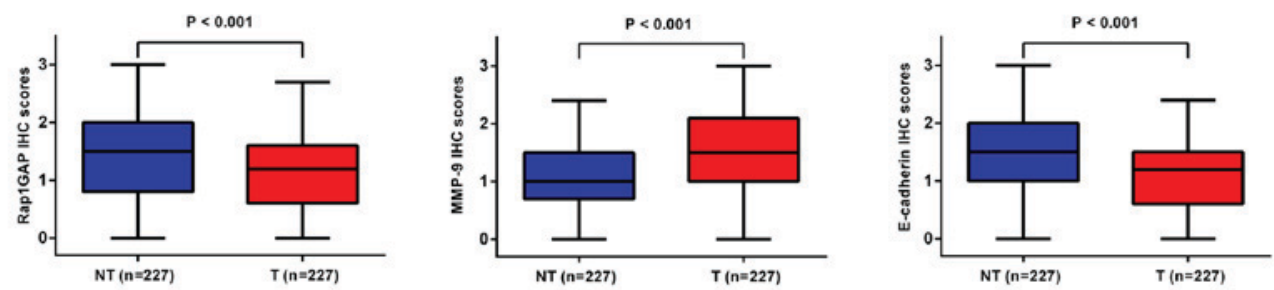

C
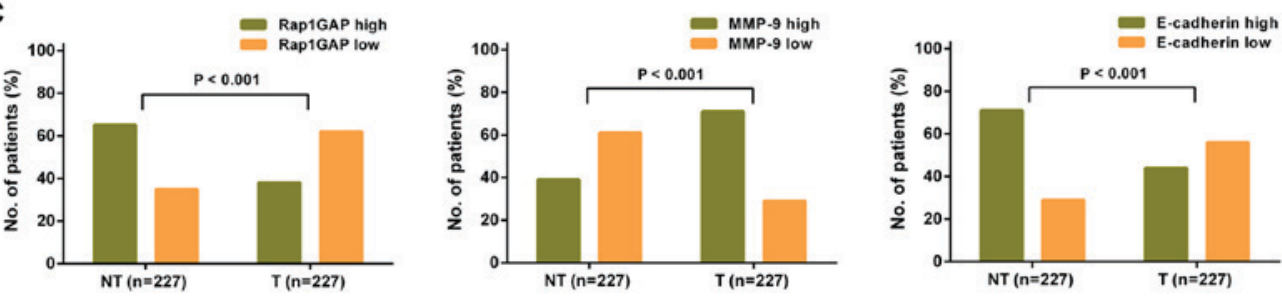

Figure 1. Rap1GAP and E-cadherin were frequently downregulated, and MMP-9 was significantly upregulated in colorectal cancer. (A) Representative immunohistochemical expression patterns of Rap1GAP (magnification x100 and x400 below), MMP-9 (magnification x200 above and x400 below) and E-cadherin in $227 \mathrm{~T}$ tissues (magnification x100 above and x400 below) and paired NT specimens (magnification x100 above and x400 below). (B) Mean \pm standard error of Rap1GAP, MMP-9 and E-cadherin expression levels were compared between T and NT specimens. (C) Percentage of patients with different expression levels of Rap1GAP, MMP-9 and E-cadherin in T or NT tissues. Rap1GAP, Rap1 GTPase-activating protein; MMP-9, matrix metallopeptidase 9; T, human primary colorectal tumor; NT, paired para-carcinoma; IHC, immunohistochemistry.
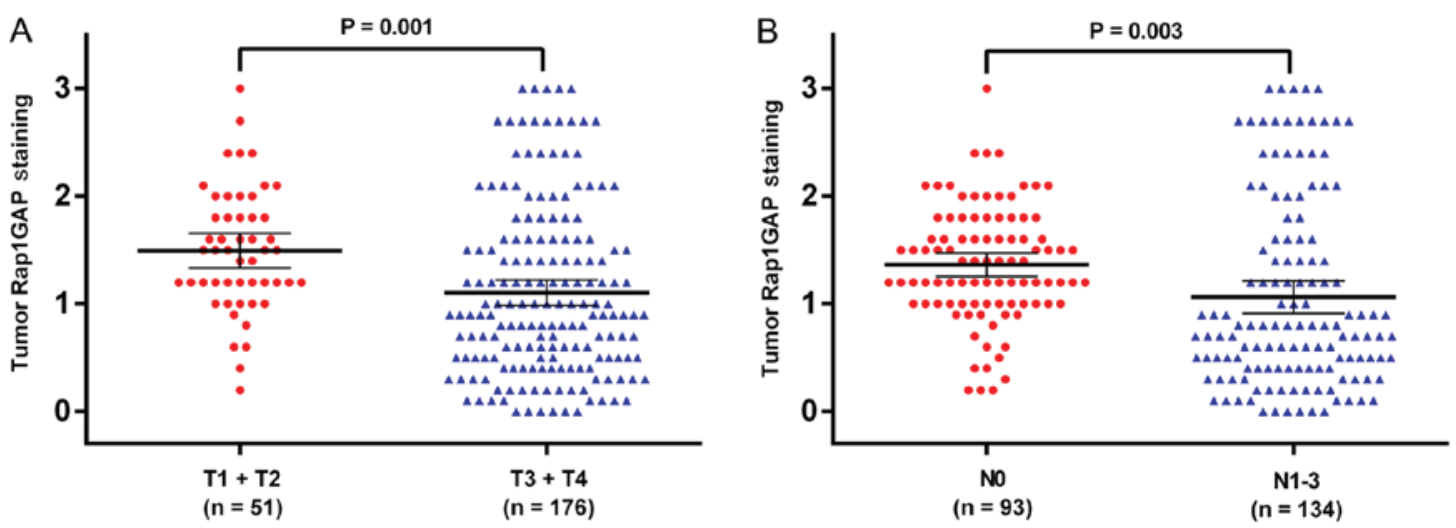

Figure 2. Comparison of Rap1GAP expression by depth of invasion and lymph node metastasis in colorectal cancer. Rap1GAP expression is notably decreased in the (A) prominent serosal invasion (T-value) and (B) lymph node metastasis groups. Rap1GAP, Rap1 GTPase-activating protein; T, tumor; N, node.

A number of studies have reported that Rap1GAP inhibits tumor cell proliferation, invasion and metastasis in various types of human cancer and, therefore, is known as a tumor suppressor gene $(10,13,16-18)$. Furthermore, the downregulation of Rap1GAP in CRC tumor cells where alterations in cell-cell and cell-matrix adhesion are early steps in tumor dissemination supports a role for Rap1GAP depletion in tumor progression (18-21). Additionally, in the present study, the expression levels of Rap1GAP were lower in patients with depth of invasion T3+T4 and lymph node metastasis N1-3 than in those with depth of invasion T1+T2 and lymph node metastasis NO, indicating higher invasive and metastasizing activity in cancer cells with a low expression of Rap1GAP. These results suggested that the decreased 

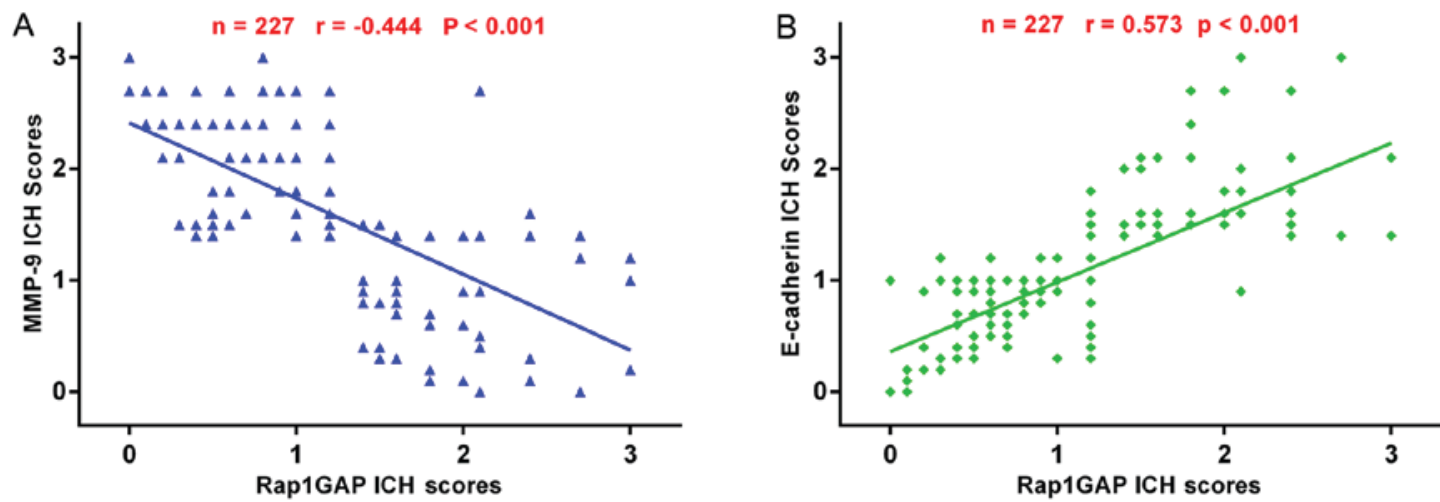

Figure 3. Correlation between the expression of Rap1GAP and that of E-cadherin or MMP-9 in 227 colorectal cancer tissues. (A) The expression of RaplGAP was negatively correlated with MMP-9 expression. (B) The expression of RaplGAP was positively correlated with E-cadherin expression. Rap1GAP, Rap1 GTPase-activating protein; MMP-9, matrix metallopeptidase 9.
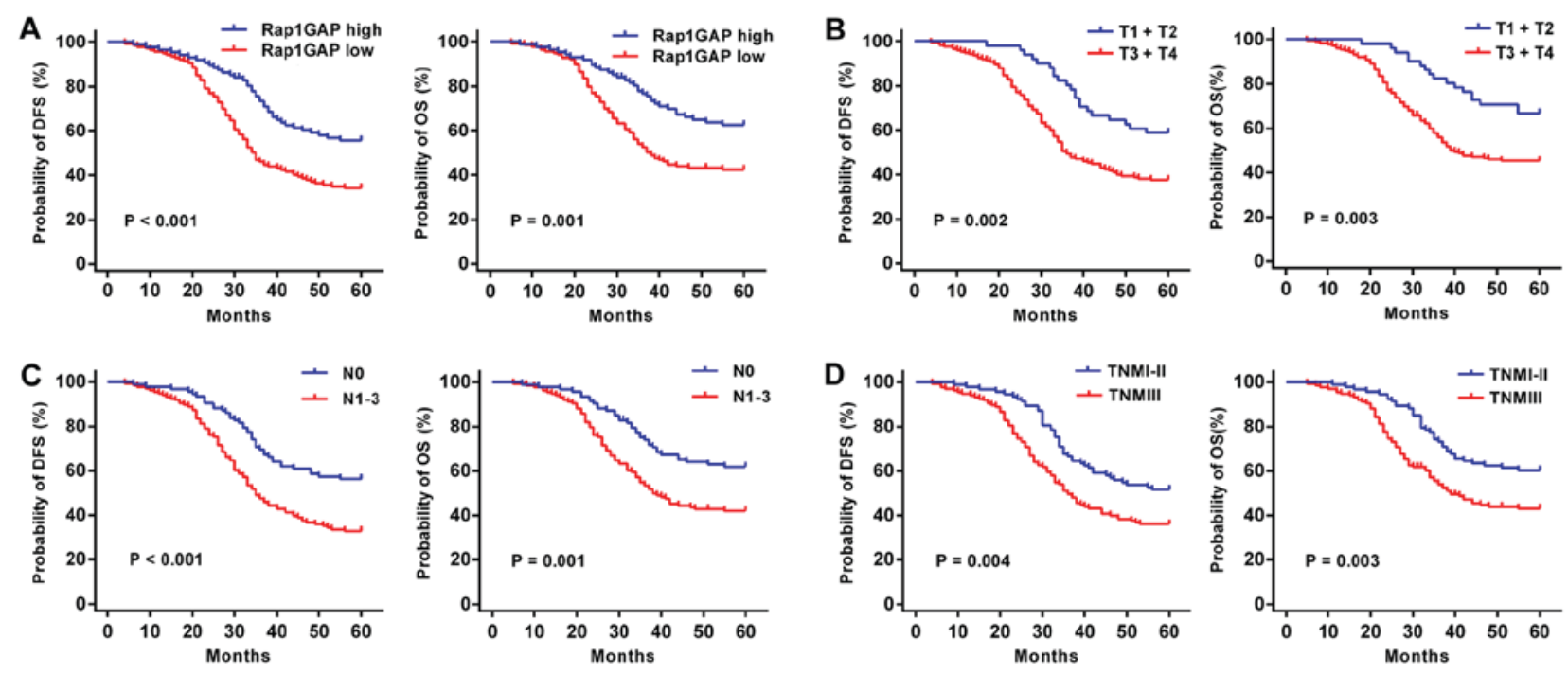

Figure 4. Kaplan-Meier survival analysis. (A) Kaplan-Meier curves for DFS and OS of patients with CRC according to Rap1GAP expression status (high vs. low expression). (B) Kaplan-Meier curves for DFS and OS of patients with CRC according to the depth of tumor invasion (T1+T2 vs. T3+T4). (C) Kaplan-Meier curves for DFS and OS of patients with CRC according to lymph node metastasis (N0 vs. N1-3). (D) Kaplan-Meier curves for DFS and OS of CRC patients according to TNM stage (TNMI-II stage or TNMIII stage). The P-value was determined using the log-rank test. DFS, disease-free survival; OS, overall survival; CRC, colorectal cancer; Rap1GAP, Rap1 GTPase-activating protein; TNM, tumor-node-metastasis.

expression of Rap1GAP may be involved in the progression of CRC.

MMPs are known as major critical molecules facilitating tumor cell invasion and metastasis by degrading the extracellular matrix (ECM) during cancer development $(22,23)$. A meta-analyses indicated that MMP-9 expression was associated with the prognosis of patients with $\mathrm{CRC}$, and that patients with higher MMP-9 expression had poorer survival rates (24). Herszényi et al (25) reported that MMP-9 may serve a role, not only in tumor invasion and initiation of metastasis, but also in carcinogenesis from colorectal adenomas. Zuzga et al (26) revealed that overexpression of MMP-9 in tumor epithelial cells was correlated with CRC metastasis. Furthermore, in head and neck squamous cell carcinoma, RaplGAP inhibited tumor cell proliferation and promoted MMP-9 secretion via Rap1-mediated effects on MMP-9 protein and mRNA (27). In leukemia, upregulation of Rap1GAP promoted NB4 and HL-60 cell differentiation and apoptosis induced by all-trans-retinoic acid (ATRA) or granulocyte and phorbol 12-myristate 13-acetate (TPA), compared with the empty vector control cells (14). In addition, the present study revealed that Rap1GAP expression was negatively correlated with MMP-9 protein expression in CRC. These results further indicate that RaplGAP may be involved in the tumorigenesis of and promote the tumor progression of CRC.

Epithelial-mesenchymal transition (EMT) is known as a crucial mechanism in tumor progression and metastasis and a key regulator of distant organ formation in CRC (28-30). EMT facilitates epithelium-derived tumor cell metastasis by promoting loss of cell adhesion and polarity, and gain of migratory and invasive properties (31). Loss of E-cadherin expression is regarded as a critical determinant in this process (32-35). Numerous studies have reported that the loss or decrease of E-cadherin expression promoted CRC invasion and metastasis and was correlated with the prognosis of patients with CRC (36-39). Furthermore, upregulation of Rap1GAP increased the expression of E-cadherin in BGC823 and SGC7901 gastric cancer cells (20). Furthermore, a significant 
positive correlation between Rap1GAP and E-cadherin in CRC was observed in the present study. These results further revealed that Rap1GAP represents a novel, selective prognostic and predictive factor that may be exploited for more effective disease stage stratification and therapeutic regimen selection in patients with CRC. Additionally, the results of the present study also suggested that decreased expression of Rap1GAP may be correlated with EMT in CRC. Therefore, future investigations should focus on the EMT molecular mechanism of the effect of Rap1GAP in CRC.

In summary, the results of the present study suggested that Rap1GAP expression is significantly and frequently downregulated in CRC, and decreased expression of Rap1GAP may serve a critical role in tumor progression and prognosis. Furthermore, decreased expression of Rap1GAP may be correlated with EMT. Notably, Rap1GAP represents a promising biomarker for prognostic prediction that may indicate treatment response and targeted therapy selection to prevent and treat metastatic disease progression in CRC. The major limitations of the present study are the relatively small patient cohort and the retrospective nature of the study. Therefore, a larger study population and a longer follow-up period are required in order to validate these results.

\section{Acknowledgements}

Not applicable.

\section{Funding}

The present study was funded by the Zhejiang Provincial Medical Science and Technology Project (grant no. 2014RCA027), and the Science and Technology Research Fund of Huzhou (grant no. 2016GY28).

\section{Availability of data and materials}

All data generated or analyzed during this study are included in this published article.

\section{Authors' contributions}

WLG designed the study, performed the major experiments and wrote the manuscript. GCY and LWL performed the sample collections and data analysis. LW participated in the experiments. All authors read and approved the final manuscript.

\section{Ethics approval and consent to participate}

The study protocols were approved by the Ethics Committee and the Human Research Review Committee of Huzhou Central Hospital and met the guidelines of the responsible governmental agency. Written informed consent was obtained from all participants.

\section{Consent for publication}

Written informed consent for publication of their data was obtained from the patients or their relatives.

\section{Competing interests}

The authors declare that they have no competing interests.

\section{References}

1. Torre LA, Bray F, Siegel RL, Ferlay J, Lortet-Tieulent J and Jemal A: Global cancer statistics, 2012. CA Cancer J Clin 65: 87-108, 2015.

2. Chen W, Zheng R, Zeng H, Zhang S and He J: Annual report on status of cancer in China, 2011. Chin J Cancer Res 27: 2-12, 2015.

3. Nagtegaal ID, Quirke P and Schmoll HJ: Has the new TNM classification for colorectal cancer improved care? Nat Rev Clin Oncol 9: 119-123, 2011.

4. Raaijmakers JH and Bos JL: Specificity in Ras and Rap signaling. J Biol Chem 284: 10995-10999, 2009.

5. Polakis P, Rubinfeld B and McCormick F: Phosphorylation of rap1GAP in vivo and by cAMP-dependent kinase and the cell cycle p34cdc2 kinase in vitro. J Biol Chem 267: 10780-10785, 1992.

6. Bos JL, de Rooij J and Reedquist KA: Rap1 signalling: Adhering to new models. Nat Rev Mol Cell Biol 2: 369-377, 2001.

7. Tsygankova OM, Feshchenko E, Klein PS and Meinkoth JL: Thyroid-stimulating hormone/cAMP and glycogen synthase kinase 3beta elicit opposing effects on Rap1GAP stability. J Biol Chem 279: 5501-5507, 2004.

8. Zhang L, Chenwei L, Mahmood R, van Golen K, Greenson J, Li G, D'Silva NJ, Li X, Burant CF, Logsdon CD and Simeone DM: Identification of a putative tumor suppressor gene RaplGAP in pancreatic cancer. Cancer Res 66: 898-906, 2006.

9. Tsygankova OM, Prendergast GV, Puttaswamy K, Wang Y, Feldman MD, Wang $H$, Brose MS and Meinkoth JL: Downregulation of Rap1GAP contributes to Ras transformation. Mol Cell Biol 27: 6647-6658, 2007.

10. Zuo H, Gandhi M, Edreira MM, Hochbaum D, Nimgaonkar VL, Zhang P, Dipaola J, Evdokimova V, Altschuler DL and Nikiforov YE: Downregulation of RaplGAP through epigenetic silencing and loss of heterozygosity promotes invasion and progression of thyroid tumors. Cancer Res 70: 1389-1397, 2010.

11. Dong X, Tang W, Stopenski S, Brose MS, Korch C and Meinkoth JL: RAP1GAP inhibits cytoskeletal remodeling and motility in thyroid cancer cells. Endocr Relat Cancer 19: 575-588, 2012.

12. Zhang Z, Mitra RS, Henson BS, Datta NS, McCauley LK, Kumar P, Lee JS, Carey TE and D'Silva NJ: Rap1GAP inhibits tumor growth in oropharyngeal squamous cell carcinoma. Am J Pathol 168: 585-596, 2006.

13. Zheng H, Gao L, Feng Y, Yuan L, Zhao H and Cornelius LA: Down-regulation of Rap1GAP via promoter hypermethylation promotes melanoma cell proliferation, survival, and migration. Cancer Res 69: 449-457, 2009.

14. Qiu T, Qi X, Cen J and Chen Z: Rap1GAP alters leukemia cell differentiation, apoptosis and invasion in vitro. Oncol Rep 28: 622-628, 2012.

15. Bailey CL, Kelly P and Casey PJ: Activation of Rap1 promotes prostate cancer metastasis. Cancer Res 69: 4962-4968, 2009.

16. Vuchak LA, Tsygankova OM and Meinkoth JL: Rap1GAP impairs cell-matrix adhesion in the absence of effects on cell-cell adhesion. Cell Adh Migr 5: 323-331, 2011.

17. Banerjee R, Mani RS, Russo N, Scanlon CS, Tsodikov A, Jing X, Cao Q, Palanisamy N, Metwally T, Inglehart RC, et al: The tumor suppressor gene rap1GAP is silenced by miR-101-mediated EZH2 overexpression in invasive squamous cell carcinoma. Oncogene 30: 4339-4349, 2011.

18. Tsygankova OM, Wang $\mathrm{H}$ and Meinkoth JL: Tumor cell migration and invasion are enhanced by depletion of Rap1 GTPase-activating protein (Rap1GAP). J Biol Chem 288: 24636-24646, 2013.

19. Tamate M, Tanaka R, Osogami H, Matsuura M, Satohisa S, Iwasaki $\mathrm{M}$ and Saito T: Rap1GAP inhibits tumor progression in endometrial cancer. Biochem Biophys Res Commun 485: 476-483, 2017.

20. Yang Y, Zhang J, Yan Y, Cai H, Li M, Sun K, Wang J, Liu X, Wang J and Duan X: Low expression of Rap1GAP is associated with epithelial-mesenchymal transition (EMT) and poor prognosis in gastric cancer. Oncotarget 8: 8057-8068, 2017.

21. Tsygankova OM, Ma C, Tang W, Korch C, Feldman MD, Lv Y, Brose MS and Meinkoth JL: Downregulation of Rap1GAP in human tumor cells alters cell/matrix and cell/cell adhesion. Mol Cell Biol 30: 3262-3274, 2010. 
22. Nelson AR, Fingleton B, Rothenberg ML and Matrisian LM Matrix metalloproteinases: Biologic activity and clinical implications. J Clin Oncol 18: 1135-1149, 2000.

23. Deryugina EI and Quigley JP: Matrix metalloproteinases and tumor metastasis. Cancer Metastasis Rev 25: 9-34, 2006.

24. Li CY, Yuan P, Lin SS, Song CF, Guan WY, Yuan L, Lai RB, Gao Y and Wang Y: Matrix metalloproteinase 9 expression and prognosis in colorectal cancer: A meta-analysis. Tumour Biol 34: 735-741, 2013

25. Herszényi L, Hritz I, Lakatos G, Varga MZ and Tulassay Z: The behavior of matrix metalloproteinases and their inhibitors in colorectal cancer. Int J Mol Sci 13: 13240-13263, 2012.

26. Zuzga DS, Gibbons AV, Li P, Lubbe WJ, Chervoneva I and Pitari GM: Overexpression of matrix metalloproteinase 9 in tumor epithelial cells correlates with colorectalcancer metastasis Clin Transl Sci 1: 136-141, 2008.

27. Mitra RS, Goto M, Lee JS, Maldonado D, Taylor JM, Pan Q, Carey TE, Bradford CR, Prince ME, Cordell KG, et al: Rap1GAP promotes invasion via induction of matrix metalloproteinase 9 secretion, which is associated with poor survival in low N-stage squamous cell carcinoma. Cancer Res 68: 3959-3969, 2008.

28. Lamouille S, Xu J and Derynck R: Molecular mechanisms of epithelial-mesenchymal transition. Nat Rev Mol Cell Biol 15: 178-196, 2014

29. Hur K, Toiyama Y, Takahashi M, Balaguer F, Nagasaka T, Koike J, Hemmi H, Koi M, Boland CR and Goel A: MicroRNA-200c modulates epithelial-to-mesenchymal transition (EMT) in human colorectal cancer metastasis. Gut 62: 1315-1326, 2013

30. Brabletz T, Hlubek F, Spaderna S, Schmalhofer O, Hiendlmeyer E, Jung A and Kirchner T: Invasion and metastasis in colorectal cancer: Epithelial-mesenchymal transition, mesenchymal-epithelial transition, stem cells and beta-catenin. Cells Tissues Organs 179: 56-65, 2005.
31. Arias AM: Epithelial mesenchymal interactions in cancer and development. Cell 105: 425-431, 2001.

32. Thiery JP, Acloque $H$, Huang RY and Nieto MA Epithelial-mesenchymal transitions in development and disease. Cell 139: 871-890, 2009.

33. Onder TT, Gupta PB, Mani SA, Yang J, Lander ES and Weinberg RA: Loss of E-cadherin promotes metastasis via multiple downstream transcriptional pathways. Cancer Res 68 3645-3654, 2008

34. Perl AK, Wilgenbus P, Dahl U, Semb H and Christofori G: A causal role for E-cadherin in the transition from adenoma to carcinoma. Nature 392: 190-193, 1998

35. Tsai JH and Yang J: Epithelial-mesenchymal plasticity in carcinoma metastasis. Genes Dev 27: 2192-2206, 2013.

36. Tsanou E, Peschos D, Batistatou A, Charalabopoulos A and Charalabopoulos K: The E-cadherin adhesion molecule and colorectal cancer. A global literature approach. Anticancer Res 28: 3815-3826, 2008

37. Kwak JM, Min BW, Lee JH, Choi JS, Lee SI, Park SS, Kim J, Um JW, Kim SH and Moon HY: The prognostic significance of Ecadherin and liver intestine-cadherin expression in colorectal cancer. Dis Colon Rectum 50: 1873-1880, 2007.

38. Hiscox S and Jiang WG: Expression of E-cadherin, alpha, beta and gamma-catenin in human colorectal cancer. Anticancer Res 17: 1349-1354, 1997.

39. Rosivatz E, Becker I, Bamba M, Schott C, Diebold J, Mayr D, Höfler $\mathrm{H}$ and Becker KF: Neoexpression of N-cadherin in E-cadherin positive colon cancers. Int J Cancer 111: 711-719, 2004.

This work is licensed under a Creative Commons Attribution-NonCommercial-NoDerivatives 4.0 International (CC BY-NC-ND 4.0) License. 